\title{
Galactic consequences of clustered star formation
}

\author{
M. R. Haas ${ }^{1}$ and P. Anders ${ }^{2}$ \\ ${ }^{1}$ Leiden Observatory, Leiden University, Postbus 9513, 2300 RA, Leiden, the Netherlands \\ email: haas@strw.leidenuniv.nl \\ ${ }^{2}$ Sterrenkundig Instituut, Utrecht University, Princetonplein 5, 3584 CC Utrecht, \\ the Netherlands \\ email: p.anders@uu.nl
}

\begin{abstract}
If all stars form in clusters and both stars and clusters follow a power-law distribution which favours the creation of low-mass objects, the numerous low-mass clusters will be deficient in high-mass stars. Therefore, the stellar mass function integrated over the entire galaxy (the integrated galactic initial mass function; IGIMF) will be steeper at the high-mass end than the underlying stellar IMF. We show how the steepness of the IGIMF depends on the sampling method and on the assumptions made regarding the star cluster mass function. We also investigate the O-star content, integrated photometry and chemical enrichment of galaxies that result from several IGIMFs compared to more standard IMFs.
\end{abstract}

Keywords. stars: luminosity function, mass function, galaxies: stellar content, galaxies: abundances, galaxies: fundamental parameters, methods: statistical

\section{Introduction}

In recent years, a series of papers (Kroupa \& Weidner 2003; Weidner \& Kroupa 2004; Weidner \& Kroupa 2005; Weidner \& Kroupa 2006: WK06) have proposed that the stellar content of an entire galaxy may not be well described by the same initial mass function (IMF) that describes the distribution of stellar masses in the star clusters, where these stars form. The reason for this is that star clusters also form with a cluster mass function $(\mathrm{CMF})$, which is a power law with an index of $\sim-2$. If the lower cluster mass limit is very low (in the mass range that is also covered by single stars, i.e., below $100 \mathrm{M}_{\odot}$, and under the assumption that the most massive star in a cluster cannot be more massive than its host cluster, $M_{\mathrm{cl}}$ ), the low-mass clusters will be deficient in high-mass stars. Therefore, if the stellar content of all clusters is added up, resulting in the integrated galactic IMF (IGIMF), the distribution of stellar masses may be steeper at the high-mass end, depending on the exact shape of the CMF.

The exact form of the IGIMF could have profound implications for the integrated properties of galaxies. High-mass stars are important, both in terms of their energy output (massive stars are bright and end their lifes in supernova explosions) and in terms of chemical enrichment. Assuming an IGIMF instead of an IMF, the supernova rate and chemical enrichment of galaxies are studied by Goodwin \& Pagel (2005) and the relation between $\mathrm{H} \alpha$ and ultraviolet luminosity in star-forming disks by PflammAltenburg \& Kroupa (2008). Recchi et al. (2009) investigates $[\alpha / \mathrm{Fe}]$ abundance ratios for IGIMFs.

In most of these studies, the assumed CMF and sampling method are kept the same. For sampling they use the 'sorted sampling' method, which was proposed by WK06 as they found it to best fit the relation between the most massive star in a cluster and the 
cluster mass. The CMF was assumed to be a pure power law with an index of -2.2 and a lower cluster mass limit of $5 \mathrm{M}_{\odot}$.

In this contribution, we vary the sampling method and CMF parameters and investigate the resulting IGIMFs. We will also estimate the observability of the effects of sampling-method and CMF variations through number counts of O-type stars, as will be observed by Gaia, the integrated photometry of distant galaxies and the chemical enrichment of galaxies (in terms of their total gas-phase metallicity).

\section{Constructing IGIMFs}

As default choice for the CMF we will use the same CMF as used in the past,

$$
\mathrm{d} N / \mathrm{d} M_{\mathrm{cl}} \propto M_{\mathrm{cl}}^{-2.2},
$$

with a lower mass limit of $5 \mathrm{M}_{\odot}$. For computational simplicity we use a Salpeter IMF for stars between 0.1 and $100 \mathrm{M}_{\odot}$.

We test several sampling methods (italic phrases refer to the legend in Figure 1):

1. Random sampling until the cluster mass is reached. As this will never reach exactly the cluster mass aimed for, we either include or exclude the last star (stop after versus stop before), only exclude it if that brings the total mass in stars closer to the cluster mass aimed for than by including it (stop nearest) or exclude the last star (which first exceeded the cluster mass) with a $50 \%$ probability (stop 50/50).

2. Sampling a specific number of stars, estimated from the total cluster mass and average mass in the IMF, with the maximum stellar mass either limited to the cluster mass or unlimited (number and number unlimited).

3. The sorted sampling method of WK06.

4. Analytical sampling, in which we assume that the IMF in a cluster is always sampled analytically, thus excluding stochastic effects.

For a more detailed description, see Haas \& Anders (2009). We use a Monte Carlo approach to reduce the numerical scatter to less than the difference between IGIMFs. We investigate the dependence of the results on the underlying IMF (we test for a Kroupatype IMF) and the results are virtually indistinguishable.

As the lack of high-mass stars is mainly caused by the low-mass clusters, the behaviour of the CMF at the low-mass end is very important. The default choice is an extrapolation of the CMF observed at higher masses $\left(M_{\mathrm{cl}} \gtrsim 10^{3} \mathrm{M}_{\odot}\right)$ down to the mass of the smallest observed star-forming clumps in the Taurus-Auriga region. We therefore test, in addition to the default choice given above, also lower limits of 1 and $50 \mathrm{M}_{\odot}$ for the clusters and power-law indices of $-1.8,-3.2$ and -4.2 (effectively changing the number of low-mass with respect to high-mass clusters).

\section{Resulting IGIMFs}

In Figure 1 we show the resulting IGIMFs from different sampling methods (left) and CMFs (right). The value of the IGIMF is divided by the value of the underlying IMF to enhance the visibility of the differences. Dotted lines indicate the slopes the different IGIMFs would have had.

It can be clearly seen that the different sampling methods give different IGIMFs. The recovered CMFs after filling the clusters with stars are indistinguishable from the input CMF. Depending on the method, the steep end has a power-law indix of about -2.6 , compared to the input value of -2.35 . The 'sorted sampling' and 'analytical sampling' methods yield even slightly steeper slopes and deviate strongly from a power-law shape. 

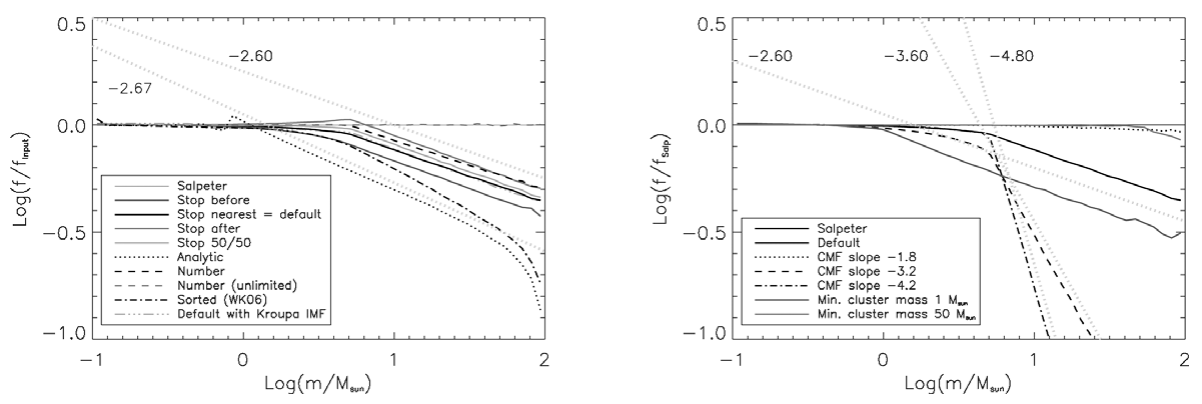

Figure 1. Resulting IGIMFs, normalized to the input IMF for different sampling methods (left) and CMFs (right). The indications correspond to the italicized descriptions in the text. The dotted lines with indices indicate the slope the respective line would have in an IGIMF.

From the Monte Carlo realisations using different CMFs, it can be seen that the slope of the CMF determines the high-mass slope of the IGIMF, while the minimum cluster mass sets the stellar mass at which the steepening sets in. If clusters do not typically go down in mass all the way to the mass range of stars, sampling effects in the IGIMF will be negligible. The low-mass end of the CMF is poorly constrained. The IGIMF could be obtained observationally, giving an indirect measure of the low-mass end of the CMF (assuming a sampling method and an underlying IMF).

\section{Galaxy properties from IGIMFs}

Using the IGIMFs as IMFs in galaxy evolution models, we can investigate the integrated properties of galaxies if their total stellar initial mass distribution is given by an IGIMF rather than a more standard IMF. The high-mass end of the stellar-mass distribution is important for galaxies, as their light in many passbands is dominated by the most massive stars for a wide range of star-formation histories. Their chemical composition depends on the metals expelled by the massive stars and the interstellar medium is strongly affected by the energy output of the massive stars exploding as supernovae.

\subsection{The number of $O$ stars in the Galaxy}

As the most pronounced effect occurs for the most massive stars, we first estimate the difference in massive-star content of the galaxies. Specifically, we estimate the number of O stars (with masses $>17 \mathrm{M}_{\odot}$ ) that Gaia will be able to observe. Assuming that GAIA will observe $10 \%$ of all $\mathrm{O}$ stars in the Galaxy, that they live for $10 \mathrm{Myr}$ and that in the past $10 \mathrm{Myr}$ the average star-formation rate of the Galaxy was $1 \mathrm{M}_{\odot} \mathrm{yr}^{-1}$, GAIA will be able to rule out several of the very extreme IGIMFs (resulting from, e.g., steep CMFs and sorted sampling) with very high significance. Judging the several different sampling methods is difficult, as the differences are on the order of $1 \sigma$, assuming purely Poissonian errors on the number counts.

If our assumptions regarding the observed fraction of $\mathrm{O}$ stars, the star-formation rate and/or the lifetimes of $\mathrm{O}$ stars are inaccurate, the resulting number of $\mathrm{O}$ stars varies linearly with either. The errors on the numbers scale with the square root of the numbers. As the numbers we calculate are on the order of 1000 to 2000 , and the differences an order of magnitude smaller, the combined effect of our assumptions may be off by a factor of a few and the differences between all IGIMFs will still be of more than $2 \sigma$ significance. 


\subsection{Photometry and chemical evolution of galaxies}

Using the GALEV evolutionary synthesis models (Bicker et al. 2004; Kotulla et al. 2009), we can assess observable properties of galaxies, in which the stellar initial mass distribution is given by IGIMFs, instead of the more popular IMFs. The GALEV models follow the chemical enrichment and photometric evolution of galaxies self-consistently in closedbox models of galaxy evolution. They can match a wide range of observed properties of galaxies of all Hubble types, such as stellar masses, star formation and multi-passband photometry.

The ingredients of the models are star-formation histories, which depend on the gas mass present (no in- or outflows are assumed, nor are they necessary), and Hubble type, spectral synthesis, and yield tables. Subsequent generations of stars are formed from the gas enriched by earlier generations.

For all Hubble types, the difference in integrated photometry in any band is smaller than the observed galaxy-to-galaxy scatter in the HYPERLEDA database (Paturel et al. 2003). Therefore, integrated photometry of galaxies will not be a discriminant between IMFs and/or IGIMFs.

Many heavy elements are mainly produced in supernova explosions of Type II (including $\mathrm{Ib}, \mathrm{c}$ ) and in the winds of massive asymptotic giant branch stars. With a deficiency in massive stars, as expected from the IGIMF, less production of metals is to be expected. The GALEV models do not follow the chemical enrichment on an element-by-element basis, but rather follow the total metallicity. Element abundances can then be obtained by assuming solar abundance ratios.

With IGIMFs as determined above, metallicities of galaxies vary by half a dex at a given gas-mass fraction. For the IGIMFs resulting from extremely steep CMFs, the difference can be up to two orders of magnitude. At given gas mass fractions, the HyperLeda database gives a scatter of about half a dex as well. Therefore, the most extreme CMFs can be ruled out by the observed gas-phase metallicities. To differentiate between several IGIMFs, more precise measurements of metallicities are necessary. The difference between sampling methods is comparable to, or slightly larger than, the spread that results from using individual metallicity measurements for the same galaxy. It is thus a possible discriminant between IGIMFs, provided that absolute determinations of gas-phase metallicities are reliable.

\section{References}

Bicker, J., Fritze-v. Alvensleben, U., Möller, C. S., \& Fricke, K. J. 2004, A\& $A, 413,37$

Goodwin, S. P. \& Pagel, B. E. J. 2005, MNRAS, 359, 707

Haas, M. R. \& Anders, P. 2009, A\&A, submitted

Kotulla, R., Fritze, U., Weilbacher, P., \& Anders, P. 2009, MNRAS, 396, 462

Kroupa, P. 2001, MNRAS, 322, 231

Kroupa, P. \& Weidner, C. 2003, ApJ, 598, 1076

Paturel, G., Petit, C., Prugniel, P., Theureau, G., Rousseau, J., Brouty, M., Dubois, P., \& Cambrésy, L. 2003, A\&\&A, 412, 45

Pflamm-Altenburg, J. \& Kroupa, P. 2008, Nature, 455, 641

Recchi, S., Calura, F., \& Kroupa, P. 2009, A\&A, 499, 711

Salpeter, E. E. 1955, ApJ, 121, 161

Weidner, C. \& Kroupa, P. 2004, MNRAS, 348, 187

Weidner, C. \& Kroupa, P. 2005, ApJ, 625, 754

Weidner, C. \& Kroupa, P. 2006, MNRAS, 365, 1333

$\dagger$ http://leda.univ-lyon1.fr/ 